Bull. Korean Math. Soc. 49 (2012), No. 2, pp. 359-365

http://dx.doi.org/10.4134/BKMS.2012.49.2.359

\title{
LIST EDGE AND LIST TOTAL COLORINGS OF PLANAR GRAPHS WITHOUT 6-CYCLES WITH CHORD
}

\author{
Aijun Dong, Guizhen Liu, and Guojun Li
}

\begin{abstract}
Giving a planar graph $G$, let $\chi_{l}^{\prime}(G)$ and $\chi_{l}^{\prime \prime}(G)$ denote the list edge chromatic number and list total chromatic number of $G$ respectively. It is proved that if a planar graph $G$ without 6-cycles with chord, then $\chi_{l}^{\prime}(G) \leq \Delta(G)+1$ and $\chi_{l}^{\prime \prime}(G) \leq \Delta(G)+2$ where $\Delta(G) \geq 6$.
\end{abstract}

\section{Introduction}

The terminology and notation used but undefined in this paper can be found in [1]. Let $G=(V, E)$ be a graph. We use $V(G), E(G), F(G), \Delta(G)$ and $\delta(G)$ to denote the vertex set, edge set, face set, maximum degree, and minimum degree of $G$, respectively. Let $d_{G}(x)$ or simply $d(x)$, denote the degree of a vertex (face) $x$ in $G$. A vertex (face) $x$ is called a $k$-vertex ( $k$-face), $k^{+}$-vertex $\left(k^{+}\right.$-face), $k^{-}$-vertex, if $d(x)=k, d(x) \geq k, d(x) \leq k$. We use $\left(d_{1}, d_{2}, \ldots, d_{n}\right)$ to denote a face $f$ if $\left(d_{1}, d_{2}, \ldots, d_{n}\right)$ are the degree of vertices incident to the face $f$. If $u_{1}, u_{2}, \ldots, u_{n}$ are the vertices on the boundary walk of a face $f$, then we write $f=u_{1} u_{2} \cdots u_{n}$. Let $\delta(f)$ denote the minimal degree of vertices incident to $f$. We use $f_{i}(v)$ denote the number of $i$-faces incident to $v$ for each $v \in V(G)$. Let $n_{i}(f)$ denote the number of $i$-vertices incident to $f$ for each $f \in F(G)$. A cycle $C$ of length $k$ is called $k$-cycle, if $x y \in E(G) \backslash E(C)$ and $x$, $y \in V(C)$, the cycle $C$ is called $k$-cycle with chord.

The mapping $L$ is said to be a total assignment for a graph $G$ if it assigns a list $L(x)$ of possible colors to each element $x \in V \cup E$. If $G$ has a proper total coloring $\phi(x) \in L(x)$ for all $x \in V \cup E$, then we say that $G$ is total-Lcolorable. Let $f: V \cup E \rightarrow N$ be a function into the positive integers. We say that $G$ is total- $f$-choosable if it is total- $L$-colorable for every total assignment $L$ satisfying $|L(x)|=f(x)$ for all $x \in V \cup E$. The list total coloring number $\chi_{l}^{\prime \prime}(G)$ of $G$ is the smallest integer $k$ such that $G$ is total- $f$-choosable when $f(x)=k$ for each $x \in V \cup E$. The list edge coloring number $\chi_{l}^{\prime}(G)$ of $G$ is

Received November 5, 2010.

2010 Mathematics Subject Classification. 05C15.

Key words and phrases. list coloring, planar graph, choosability.

This work was supported by the National Science Foundation of China $(264062$, 202799, 269960). 
defined similarly in terms of coloring edges alone; and so are the concept of edge-f-choosable. If $G$ is a graph that can not be edge- $(\Delta(G)+1)$-choosable or total- $(\Delta(G)+2)$-choosable graph with the fewest vertices and edges, then we call it a critical graph. On the list coloring number of a graph $G$, there is a famous conjecture known as the List Coloring Conjecture.

Conjecture 1. For a multigraph $G$,

$$
\text { (a) } \chi_{l}^{\prime}(G)=\chi^{\prime}(G) \text {; (b) } \chi_{l}^{\prime \prime}(G)=\chi^{\prime \prime}(G) \text {. }
$$

Part (a) of the above conjecture was formulated independently by Vizing, by Gupta, by Alberson and Collins, and by Bollobás and Harris [5, 11]. It is well known as the List Coloring Conjecture. Part (b) was formulated by Borodin, Kostochka and Woodall [2]. Part (a) and Part (b) has been proved for outerplanar graphs [13], and graphs with $\Delta \geq 12$ which can be embedded in a surface of nonnegative characteristic [2]. List Coloring Conjecture has been proved for a few other special graphs, such as bipartite multigraphs [4], complete graphs of odd order [6]. There are several related results for planar graphs without some short cycles or by adding girth restrictions $[7,8,9,3,16$, $14,15,10]$.

In this paper, we shall show that if $G$ is a planar graph without 6-cycles with chord, then $\chi_{l}^{\prime}(G) \leq \Delta(G)+1$ and $\chi_{l}^{\prime \prime}(G) \leq \Delta(G)+2$ where $\Delta(G) \geq 6$.

\section{Planar graphs without 6-cycles with chord}

First let us introduce an important lemma.

Lemma 2.1. Let $G$ be a critical planar graph without 6-cycles with chord. If $\Delta(G) \geq 6$, then there is an edge uv $\in E(G)$ such that $\min \{d(u), d(v)\} \leq$ $\left\lfloor\frac{\Delta(G)+1}{2}\right\rfloor$ and $d(u)+d(v) \leq \max \{8, \Delta(G)+2\}$.

Proof. For $G$ is a critical planar graph with $\Delta(G) \geq 6$, then $G$ contains no $\left(4,4,5^{-}\right)$-face $f=u v w$. By contradiction, let $L^{\prime}$ and $L^{\prime \prime}$ be any list assignments such that $\left|L^{\prime}(e)\right|=\Delta(G)+1$ for each $e \in E(G)$ and $\left|L^{\prime \prime}(x)\right|=\Delta(G)+2$ for each $x \in V(G) \cup E(G)$.

Let $G^{\prime}=G-\{u v, v w, w u\}$. By $G$ is a critical graph, $G^{\prime}$ is edge- $L^{\prime}$-colorable. Now there are at least three colors available for $u v$, and at least two colors for $v w$ and $w u$. We can easily color $v w, u w$, and $u v$ successively. So $G$ is edge- $L^{\prime}$-colorable, a contradiction.

For the same reason, $G^{\prime}$ is total- $L^{\prime \prime}$-colorable. Erase the colors of the vertices $u, v, w$. For each element $x$ incident with $f$, we define a reduced total list $\bar{L}^{\prime \prime}(x)$ such that $\overline{L^{\prime \prime}}(x)=L^{\prime \prime}(x) \backslash\left\{\phi\left(x^{\prime}\right) \mid x^{\prime}\right.$ is incident with or adjacent to $x$, and, $x^{\prime}$ is not incident with $\left.f\right\}$ where $\phi\left(x^{\prime}\right)$ denotes the color of the element $x^{\prime}$. Then $\left|\overline{L^{\prime \prime}}(u)\right| \geq 4,\left|\overline{L^{\prime \prime}}(v)\right| \geq 4,\left|\overline{L^{\prime \prime}}(w)\right| \geq 2,\left|\overline{L^{\prime \prime}}(u v)\right| \geq 4,\left|\overline{L^{\prime \prime}}(u w)\right| \geq 3$, $\left|\overline{L^{\prime \prime}}(v w)\right| \geq 3$. If there is a color $\alpha \in \overline{L^{\prime \prime}}(u w) \backslash \overline{L^{\prime \prime}}(u)$, then we can color $u w$ with the color $\alpha$, and color $w, w v, v, u v$, and $u$ successively. So $\overline{L^{\prime \prime}}(u w) \subseteq \overline{L^{\prime \prime}}(u)$. Similarly, $\overline{L^{\prime \prime}}(v w) \subseteq \overline{L^{\prime \prime}}(v)$. If there is a color $\beta \in \overline{L^{\prime \prime}}(u) \backslash \overline{L^{\prime \prime}}(v)$, then we can 
color $u$ with $\beta$, and color $w, u w, v w, u v$ and $v$ successively. So $\overline{L^{\prime \prime}}(u)=\overline{L^{\prime \prime}}(v)$. Thus there is a color $\gamma \in \overline{L^{\prime \prime}}(u w) \bigcap \overline{L^{\prime \prime}}(v)$. We color $u w$ and $v$ with $\gamma$, and color $w, v w, u v$ and $u$ successively. From the above discussion, in any case, $f$ is total- $\bar{L}^{\prime \prime}$-colorable. So $G$ is total- $L^{\prime \prime}$-colorable, a contradiction.

In the following, we show that for the critical planar graph without 6cycles with chord, if $\Delta(G) \geq 6$, then there is an edge $u v \in E(G)$ such that $\min \{d(u), d(v)\} \leq\left\lfloor\frac{\Delta(G)+1}{2}\right\rfloor$ and $d(u)+d(v) \leq \max \{8, \Delta(G)+2\}$. By contradiction, we have $d(u)+d(v) \geq \max \{9, \Delta(G)+3\}$ for each edge $u v \in E(G)$ such that $\min \{d(u), d(v)\} \leq\left\lfloor\frac{\Delta(G)+1}{2}\right\rfloor$. It is clear that $\delta(G) \geq 3$.

By Euler's formula $|V|-|E|+|F|=2$ and $\sum_{v \in V(G)} d(v)=\sum_{f \in F(G)} d(f)=$ $2|E|$, we have

$$
\sum_{v \in V(G)}(2 d(v)-6)+\sum_{f \in F(G)}(d(f)-6)=-6(|V|-|E|+|F|)=-12 .
$$

Define an initial charge function $w$ on $V(G) \cup F(G)$ by setting $w(v)=2 d(v)-$ 6 if $v \in V(G)$ and $w(f)=d(f)-6$ if $f \in F(G)$, so that $\sum_{x \in V(G) \cup F(G)} W(x)=$ -12 . Now redistribute the charge according to the following discharging rules.

For convenience, let $w \overline{(v)}$ denote the total charge transferred from a vertex $v$ to all its incident 4 - and 5-faces where $d(v)=4$.

$D 1$. If $f$ is a 3 -face incident with a vertex $v$, then $v$ gives $f$ charge $\frac{2-w \overline{(v)}}{f_{3}(v)}$ if $d(v)=4, \frac{4}{3}$ if $d(v)=5, \frac{3}{2}$ if $d(v) \geq 6$.

$D 2$. If $f$ is a 4 -face incident with a vertex $v$, then $v$ gives $f$ charge $\frac{1}{2}$ if $d(v)=4$ or 5,1 if $d(v) \geq 6$.

$D 3$. If $f$ is a 5 -face incident with a vertex $v$, then $v$ gives $f$ charge $\frac{1}{5}$ if $d(v)=4$ or $5, \frac{1}{3}$ if $d(v) \geq 6$.

Let the new charge of each element $x$ be $w^{\prime}(x)$ for each $x \in V(G) \cup F(G)$.

In the following, let us check the new charge of each element $x \in V(G) \cup$ $F(G)$.

Suppose $d(v)=3$. Then $w^{\prime}(v)=w(v)=0$.

Suppose $d(v)=4$. Then $w(v)=2, f_{3}(v) \leq 4$. If $f_{3}(v) \geq 1$, then $w^{\prime}(v) \geq$ $2-\frac{2-w(v)}{f_{3}(v)} f_{3}(v)-w \overline{(v)}=0$ by $D 1$. Otherwise, i.e., $f_{3}(v)=0$, then $f_{4}(v) \leq 2$, $f_{5}(v) \leq 4$ for $G$ contains no 6 -cycles with chord. We have $w^{\prime}(v)>2-\frac{1}{2} \times 2-$ $\frac{1}{5} \times 4=\frac{1}{5}>0$ by $D 2$ and $D 3$.

Suppose $d(v)=5$. Then $w(v)=4, f_{3}(v) \leq 3$ for $G$ contains no 6-cycles with chord. If $f_{3}(v)=3$, then $f_{4}(v)=0$ and $f_{5}(v)=0$ for $G$ contains no 6-cycles with chord. We can get $w^{\prime}(v) \geq 4-\frac{4}{3} \times 3=0$ by $D 1$. If $f_{3}(v)=2$, then $f_{4}(v) \leq 1$ and $f_{5}(v) \leq 1$ for $G$ contains no 6 -cycles with chord. We can get $w^{\prime}(v) \geq 4-\frac{4}{3} \times 2-\frac{1}{2}-\frac{1}{5}=\frac{19}{30}>0$ by $D 1, D 2$ and $D 3$. If $f_{3}(v)=1$, then $f_{4}(v) \leq 2$ and $f_{5}(v) \leq 2$ for $G$ contains no 6 -cycles with chord. We can get $w^{\prime}(v) \geq 4-\frac{4}{3}-\frac{1}{2} \times 2-\frac{1}{5} \times 2=\frac{19}{15}>0$ by $D 1, D 2$ and $D 3$. If $f_{3}(v)=0$, 
then $f_{4}(v) \leq 2$ or $f_{5}(v) \leq 5$ for $G$ contains no 6 -cycles with chord, we have $w^{\prime}(v)>4-\frac{1}{2} \times 2-\frac{1}{5} \times 5=2>0$ by $D 2$ and $D 3$.

Suppose $d(v)=6$. Then $w(v)=6, f_{3}(v) \leq 4$ for $G$ contains no 6 -cycles with chord. If $f_{3}(v)=4$, then $f_{4}(v)=0$ and $f_{5}(v)=0$ for $G$ contains no 6-cycles with chord. We can get $w^{\prime}(v) \geq 6-\frac{3}{2} \times 4=0$ by $D 1$. If $f_{3}(v)=3$, then $f_{4}(v) \leq 1$ and $f_{5}(v) \leq 1$ for $G$ contains no 6 -cycles with chord. We can get $w^{\prime}(v) \geq 6-\frac{3}{2} \times 3-1-\frac{1}{3}=\frac{1}{6}>0$ by $D 1, D 2$ and $D 3$. If $f_{3}(v)=2$, then $f_{4}(v) \leq 2$ and $f_{5}(v) \leq 2$ for $G$ contains no 6 -cycles with chord. We can get $w^{\prime}(v) \geq 6-\frac{3}{2} \times 2-1 \times 2-\frac{1}{3} \times 2=\frac{1}{3}>0$ by D1, D2 and D3. If $f_{3}(v)=1$, then $f_{4}(v) \leq 3$ and $f_{5}(v) \leq 3$ for $G$ contains no 6 -cycles with chord. We can get $w^{\prime}(v) \geq 6-\frac{3}{2}-1 \times 3-\frac{1}{3} \times 3=\frac{1}{2}>0$ by D1, D2 and D3. If $f_{3}(v)=0$, then $f_{4}(v) \leq 3$ or $f_{5}(v) \leq 6$ for $G$ contains no 6 -cycles with chord, we have $w^{\prime}(v)>6-1 \times 3-\frac{1}{3} \times 6=1>0$ by $D 2$ and $D 3$.

Suppose $d(v)=7$. Then $w(v)=8, f_{3}(v) \leq 5$ for $G$ contains no 6 -cycles with chord. If $f_{3}(v)=5$, then $f_{4}(v)=0$ and $f_{5}(v)=0$ for $G$ contains no 6 -cycles with chord. We can get $w^{\prime}(v) \geq 8-\frac{3}{2} \times 5=\frac{1}{2}>0$ by $D 1$. If $f_{3}(v)=4$, then $f_{4}(v) \leq 1$ and $f_{5}(v)=0$ for $G$ contains no 6 -cycles with chord. We can get $w^{\prime}(v) \geq 8-\frac{3}{2} \times 4-1=1>0$ by $D 1$ and $D 2$. If $f_{3}(v)=3$, then $f_{4}(v) \leq 2$ and $f_{5}(v) \leq 2$ for $G$ contains no 6 -cycles with chord. We can get $w^{\prime}(v) \geq 8-\frac{3}{2} \times 3-1 \times 2-\frac{1}{3} \times 2=\frac{5}{6}>0$ by $D 1, D 2$ and D3. If $f_{3}(v)=2$, then $f_{4}(v) \leq 3$ and $f_{5}(v) \leq 3$ for $G$ contains no 6 -cycles with chord. We can get $w^{\prime}(v) \geq 8-\frac{3}{2} \times 2-1 \times 3-\frac{1}{3} \times 3=1>0$ by $D 1, D 2$ and $D 3$. If $f_{3}(v) \leq 1$, then $f_{4}(v) \leq 3$ or $f_{5}(v) \leq 7$ for $G$ contains no 6 -cycles with chord, we have $w^{\prime}(v)>8-\frac{3}{2}-1 \times 3-\frac{1}{3} \times 7=\frac{7}{6}>0$ by $D 1, D 2$ and $D 3$.

Suppose $d(v)=8$. Then $w(v)=10, f_{3}(v) \leq 6$ for $G$ contains no 6 -cycles with chord. If $f_{3}(v)=6$ or 5 , then $f_{4}(v)=0$ and $f_{5}(v)=0$ for $G$ contains no 6 -cycles with chord. We can get $w^{\prime}(v) \geq 10-\frac{3}{2} \times 6=1>0$ by $D 1$. If $f_{3}(v)=4$, then $f_{4}(v) \leq 1$ and $f_{5}(v) \leq 1$ for $G$ contains no 6 -cycles with chord. We can get $w^{\prime}(v) \geq 10-\frac{3}{2} \times 4-1-\frac{1}{3}=\frac{8}{3}>0$ by $D 1, D 2$ and $D 3$. If $f_{3}(v)=3$, then $f_{4}(v) \leq 2$ and $f_{5}(v) \leq 3$ for $G$ contains no 6 -cycles with chord. We can get $w^{\prime}(v) \geq 10-\frac{3}{2} \times 3-1 \times 2-\frac{1}{3} \times 3=\frac{5}{2}>0$ by $D 1, D 2$ and $D 3$. If $f_{3}(v)=2$, then $f_{4}(v) \leq 4$ and $f_{5}(v) \leq 4$ for $G$ contains no 6 -cycles with chord. We can get $w^{\prime}(v) \geq 10-\frac{3}{2} \times 2-1 \times 4-\frac{1}{3} \times 4=\frac{5}{3}>0$ by $D 1, D 2$ and $D 3$. If $f_{3}(v) \leq 1$, then $f_{4}(v) \leq 4$ or $f_{5}(v) \leq 8$ for $G$ contains no 6 -cycles with chord, we have $w^{\prime}(v)>10-\frac{3}{2}-1 \times 4-\frac{1}{3} \times 8=\frac{11}{6}>0$ by $D 1, D 2$ and $D 3$.

Suppose $d(v) \geq 9$. Then $w(v)=2 d(v)-6, f_{4}(v) \leq d(v)-\frac{4}{3} \times f_{3}(v)$, $f_{5}(v) \leq d(v)-\frac{4}{3} f_{3}(v)$ for $G$ contains no 6-cycle with chord. So $w^{\prime}(v) \geq$ $2 d(v)-6-\frac{3}{2} f_{3}(v)-f_{4}(v)-\frac{1}{3} f_{5}(v) \geq 2 d(v)-6-\frac{3}{2} f_{3}(v)-d(v)+\frac{4}{3} f_{3}(v)-$ $\frac{1}{3} d(v)+\frac{4}{9} f_{3}(v) \geq \frac{2}{3} d(v)-6+\frac{5}{18} f_{3}(v)$ by $D 1, D 2$ and $D 3$. For $0 \leq f_{3}(v)$, we have $w^{\prime}(v) \geq \frac{2}{3} d(v)-6 \geq 0$.

Suppose $d(f)=3$. Then $w(f)=-3$.

Case 1. $\delta(f)=3$, then $f$ is a $\left(3,6^{+}, 6^{+}\right)$-face by assumption. We have $w^{\prime}(f)=-3+\frac{3}{2} \times 2=0$ by $D 1$. 
Case 2. $\delta(f)=4$, then $f$ is a $\left(4,4,6^{+}\right)$- or $\left(4,5^{+}, 5^{+}\right)$-face for $G$ contains no $\left(4,4,5^{-}\right)$-face.

Case 2.1. $f$ is a $\left(4,4,6^{+}\right)$-face. For convenience, let $f=u v w$ where $d(u)=$ $d(v)=4$. If one of the 4 -vertex is incident to at least three 3 -faces, without loss of generality, let $f_{3}(u) \geq 3$, then $f_{4}(u)=f_{5}(u)=0$ and $f_{3}(v)+f_{4}(v)+f_{5}(v) \leq 2$ for $G$ contains no 6-cycles with chord. We have $w^{\prime}(f) \geq-3+\frac{1}{2}+\frac{3}{2}+\frac{2-w(v)}{f_{3}(v)} \geq$ $-3+\frac{1}{2}+\frac{3}{2}+1=0$ by $D 1, D 2$ and $D 3$. Otherwise, i.e., $f_{3}(u) \leq 2, f_{3}(v) \leq 2$, then we have $\frac{2-w \bar{v})}{f_{3}(v)} \geq \frac{4}{5}$ for $G$ contains no 6 -cycles with chord and by $D 1, D 2$ and $D 3$. So $w^{\prime}(f) \geq-3+\frac{4}{5} \times 2+\frac{3}{2}=\frac{1}{10}>0$ by $D 1$.

Case 2.2. $f$ is a $\left(4,5^{+}, 5^{+}\right)$-face. By $D 1, D 2$ and $D 3$, we have $\frac{2-w(v)}{f_{3}(v)} \geq \frac{1}{2}$. So $w^{\prime}(f) \geq-3+\frac{1}{2}+\frac{4}{3} \times 2=\frac{1}{6}>0$ by $D 1$.

Case 3. $\delta(f) \geq 5$, then we have $w^{\prime}(f)=-3+\frac{4}{3} \times 3=1>0$ by $D 1$.

Suppose $d(f)=4$. Then $w(f)=-2$. If $\delta(f)=3$, then $f$ is a $\left(3,3^{+}, 6^{+}, 6^{+}\right)$face by assumption. We have $w^{\prime}(f) \geq-2+1 \times 2=0$ by $D 2$. If $\delta(f) \leq 4$, then $f$ is a $\left(4^{+}, 4^{+}, 4^{+}, 4^{+}\right)$-face. We have $w^{\prime}(f) \geq-2+\frac{1}{2} \times 4=0$ by $D 2$.

Suppose $d(f)=5$. Then $w(f)=-1$.

Case 4. $\delta(f)=3$, then $n_{3}(f) \leq 2$ by assumption. If $n_{3}(f)=2$, then $f$ is a $\left(3,3,6^{+}, 6^{+}, 6^{+}\right)$-face by assumption. We have $w^{\prime}(f) \geq-1+\frac{1}{3} \times 3=0$ by $D 3$. If $n_{3}(f)=1$, then $f$ is a $\left(3,4^{+}, 4^{+}, 6^{+}, 6^{+}\right)$-face by assumption. We have $w^{\prime}(f) \geq-1+\frac{1}{3} \times 2+\frac{1}{5} \times 2=\frac{1}{15}>0$ by $D 3$.

Case 5. $\delta(f)=4$, then we have $w^{\prime}(f) \geq-1+\frac{1}{5} \times 5=0$ by $D 3$.

Suppose $d(f) \geq 6$. Then $w^{\prime}(f)=w(f) \geq 0$.

From the above discussion, we can obtain $\sum_{x \in V(G) \cup F(G)} w^{\prime}(x) \geq 0>-12$, a contradiction.

In the following, let us give the proof of the main theorem.

Theorem 2.2. If $G$ is a planar graph without 6 -cycles with chord, then $\chi_{l}^{\prime}(G) \leq$ $\Delta(G)+1$ and $\chi_{l}^{\prime \prime}(G) \leq \Delta(G)+2$ where $\Delta(G) \geq 6$.

Proof. By contradiction, let $G^{\prime}$ and $G^{\prime \prime}$ be minimal counterexamples (i.e., critical graphs) to the conclusions for $\chi_{l}^{\prime}$ and $\chi_{l}^{\prime \prime}$ respectively, and let $L^{\prime}$ and $L^{\prime \prime}$ be list assignments such that $\left|L^{\prime}(e)\right|=\Delta+1$ for each $e \in E(G)$ and $G^{\prime}$ is not edge- $L^{\prime}$-colorable, and $\left|L^{\prime \prime}(x)\right|=\Delta+2$ for each $x \in V(G) \cup E(G)$ and $G^{\prime \prime}$ is not total- $L^{\prime \prime}$-colorable. By Lemma 2.1, $G^{\prime}$ and $G^{\prime \prime}$ contains an edge $u v \in E(G)$ such that $\min \{d(u), d(v)\} \leq\left\lfloor\frac{\Delta(G)+1}{2}\right\rfloor$ and $d(u)+d(v) \leq \max \{8, \Delta(G)+2\}$.

Let $\bar{G}^{\prime}=G^{\prime}-u v$. Then $\bar{G}^{\prime}$ is edge- $L^{\prime}$-colorable by assumption. For $d(u)+$ $d(v) \leq \max \{8, \Delta(G)+2\}$, there is at most $\Delta(G)$ edges which are adjacent with $u v$ in $\bar{G}^{\prime}$. So there is at least one color in $L^{\prime}(u v)$ which we can use to color $u v$. Then $G^{\prime}$ is edge- $L^{\prime}$-colorable, a contradiction.

Let $\bar{G}^{\prime \prime}=G^{\prime \prime}-u v$. Then $\bar{G}^{\prime \prime}$ is total- $L^{\prime \prime}$-colorable by assumption. Without loss of generality, let $d(u)=\min \{d(u), d(v)\}$. Erase the color of $u$, then there 
is at least one color in $L^{\prime \prime}(u v)$ which we can use to color $u v$ for $d(u)+d(v) \leq$ $\max \{8, \Delta(G)+2\}$. For $d(u) \leq\left\lfloor\frac{\Delta(G)+1}{2}\right\rfloor$, then $u$ is adjacent to at most $\left\lfloor\frac{\Delta(G)+1}{2}\right\rfloor$ vertices or is incident to $\left\lfloor\frac{\Delta(G)+1}{2}\right\rfloor$ edges. So there is at least one color in $L^{\prime}(u)$ which we can use to color $u$. Then $G^{\prime \prime}$ is total- $L^{\prime \prime}$-colorable, a contradiction. So $\chi_{l}^{\prime}(G) \leq \Delta(G)+1$ and $\chi_{l}^{\prime \prime}(G) \leq \Delta(G)+2$

\section{References}

[1] J. A. Bondy and U. S. R. Murty, Graph Theory with Applications, North-Holland, New York, 1976.

[2] O. Borodin, A. Kostochka, and D. Woodall, List edge and list total colourings of multigraphs, J. Combin. Theory Ser. B 71 (1997), no. 2, 184-204.

[3] J. Cai, J. Hou, X. Zhang, and G. Liu, Edge-choosability of planar graphs without noninduced 5-cycles, Inform. Process. Lett. 109 (2009), no. 7, 343-346.

[4] F. Galvin, The list chromatic index of a bipartite multigraph, J. Combin. Theory Ser. B 63 (1995), no. 1, 153-158.

[5] R. Häggkvist and A. Chetwynd, Some upper bounds on the total and list chromatic numbers of multigraphs, J. Graph Theory 16 (1992), no. 5, 503-516.

[6] R. Häggkvist and J. Janssen, New bounds on the list-chromatic index of the complete graph and other simple graphs, Combin. Probab. Comput. 6 (1997), no. 3, 295-313.

[7] J. Hou, G. Liu, and J. Cai, Edge-choosability of planar graphs without adjacent triangles or without 7-cycle, Discrete Math. 309 (2009), no. 1, 77-84.

[8] - List edge and list total colorings of planar graphs without 4-cycles, Theoret. Comput. Sci. 369 (2006), no. 1-3, 250-255.

[9] _ List edge and list total colorings of planar graphs without short cycles, Inform. Process. Lett. 108 (2008), no. 6, 347-351.

[10] J. Hou, G. Liu, and J. Wu, Some results on list total colorings of planar graphs, Lecture Note in Computer Science 4489 (2007), 320-328.

[11] T. Jensen, B. Toft, Graph Coloring Problem, Wiley-Interscience, New York, 1995.

[12] A. Kostochka, List edge chromatic number of graphs with large girth, Discrete Math. 101 (1992), no. 1-3, 189-201.

[13] W. Wang and K. Lih, Choosability, edge-choosability and total choosability of outerplane graphs, European J. Combin. 22 (2001), no. 1, 71-78.

[14] Choosability and edge choosability of planar graphs without five cycles, Appl. Math. Lett. 15 (2002), no. 5, 561-565.

[15] Structural properties and edge choosability of planar graphs without 6-cycles, Combin. Probab. Comput. 10 (2001), no. 3, 267-276.

[16] L. Zhang and B. Wu, Edge choosability of planar graphs without small cycles, Discrete Math. 283 (2004), no. 1-3, 289-293.

Aijun Dong

SChool of Mathematics

SHANDONG UNIVERSITY

JinAN, 250100, P. R. ChinA

E-mail address: dongaijun@mail.sdu.edu.cn 


\section{Guizhen LiU}

SCHOOL OF Mathematics

SHANDONG UNIVERSITY

JinAN, 250100, P. R. CHINA

E-mail address: gzliu@sdu.edu.cn

GUOJUn Li

School of Mathematics

SHANDONG UNIVERSITY

JinAN, 250100, P. R. CHINA

E-mail address: gj1@sdu.edu.cn 